\title{
Less-invasive tools and technique for fully magnetically levitated centrifugal pump implantation
}

\author{
Ivan Netuka, Miroslav Konarik, Martin Pokorny, Peter Ivak, Jiri Maly, Ondrej Szarszoi \\ Department of Cardiovascular Surgery, Institute for Clinical and Experimental Medicine, Prague, Czech Republic \\ Correspondence to: Ivan Netuka. Department of Cardiovascular Surgery, Institute for Clinical and Experimental Medicine, Videnska 1958/9, 140 21 \\ Prague 4, Czech Republic. Email: ivan.netuka@ikem.cz.
}

Submitted Dec 09, 2020. Accepted for publication Jan 18, 2021.

doi: 10.21037/acs-2020-cfmcs-32

View this article at: http://dx.doi.org/10.21037/acs-2020-cfmcs-32

\section{Clinical vignette}

A sixty-year-old man with acute myocardial infarction and developing cardiogenic shock was admitted for emergency coronary angiography, revealing $30 \%$ left main stenosis, $90 \%$ left anterior descending artery (LAD) stenosis and $50 \%$ circumflex (LCX) stenosis. Successful dilatation of the LAD/LCX with drug-eluting stents (kissing stent technique) was accomplished via percutaneous coronary intervention, followed by Impella CP percutaneous assist device (Abiomed US, Danvers, Massachusetts, USA) insertion due to progressive hemodynamic instability. Severe left ventricular (LV) dysfunction with an ejection fraction of $25 \%$ persisted over the next twelve days with failure to wean the patient from Impella CP, despite combined inotropic support, including levosimendan. Given the insufficient LV recovery, contrasted with only moderate right ventricular dysfunction, and the absence of concomitant valvulopathies, the patient was scheduled for a less invasive $L V$ assist device implantation (HeartMate 3, Abbott, Abbott Park, IL, USA). The patient was discharged twenty-one days post-implant.

\section{Surgical techniques}

\section{Preparation}

The patient is placed in the supine position and a surgical pad placed underneath the left hemithorax to spread the left-sided intercostal spaces, facilitating ventricular apex exposure. Once optimal positioning is achieved, an appropriate intercostal space to approach the apex is marked by transthoracic echocardiography. The patient is then draped using standard sterile conditions from sternum to thighs to allow bilateral groin exposure.

\section{Exposition}

Preventative measures addressing potential hemodynamic instability and expedited extracorporeal circulation should include venous cannulation guidewire insertion under transesophageal echocardiography guidance and arterial femoral access. The left anterior thoracotomy is typically from the fifth or sixth intercostal space given previous echocardiography-based visualization of the heart. The ascending aorta is exposed through an upper J-hemisternotomy, or alternatively, right-sided second intercostal space thoracotomy.

\section{Operation}

The pectoral and intercostal muscles are transected, a soft tissue retractor inserted, and the pericardial sac opened. Selective bronchial intubation with the left lung collapsed is recommended to facilitate exposure of the apex prior to commencing cardiopulmonary bypass (CPB). Both the 'first core then sew' or the opposite, 'first sew then core' techniques may be used. In the latter technique, the cuff is secured in the apical position by two Teflon-felt pledgeted polypropylene monofilament 3/0 sutures in contralateral segments and subsequently affixed by an identical nontransmural running suture without additional felt strips. Prophylactic use of surgical glue may be advisable.

The outflow graft affixed to the pump is tunneled through the pericardium, trimmed in length and sutured to the ascending aorta. After full heparinization, the side-biting clamp is removed. Arterial cannula to the ascending aorta, and the venous cannula via the femoral vein are inserted. $\mathrm{CPB}$ is commenced and Impella $\mathrm{CP}$ 
retracted to the descending aorta while both carotid arteries are manually compressed to mitigate the risk of cerebral thromboembolism. Once the left ventricular (LV) apex is cored and inspected, the device is passed through the intercostal space, facilitated by a proprietary clamp (Omnimedics CZ, Ltd., Prague, Czech Republic), and the pump inflow cannula is inserted. To reduce the pump profile during insertion, the sliding lock should be kept closed during pump deployment until the pump is completely engaged into the apical cuff. Subsequently, it can be reopened and secured in the final position. Due to the limited intercostal space for pump insertion, a semirigid bend relief should be kept on the graft unattached to the pump body. The outflow graft is positioned parallel to the diaphragm and along the right ventricle (RV) and lateral side of the right atrium upwards to the ascending aorta. This configuration eliminates compression of the RV by the graft, as well as potential for direct damage at the time of heart transplant. Finally, the bend relief is affixed using the second dedicated proprietary clamp (Omnimedics CZ, Ltd.), providing a strong connection to the pump body. The driveline is then tunneled through the rectus abdominis muscle, externalized, and the pump is started. Upon meticulous de-airing through the outflow graft, the heartlung machine is weaned with CPB total run-time of fifteen minutes.

\section{Completion}

Despite preserved pericardial restraint with the lessinvasive implant, which optimizes physiological boundaries of right ventricular performance, our unit protocol mandates inhaled nitric oxide in the early postoperative period. Once acceptable hemodynamics and hemostasis following protamine administration are accomplished, the pump is isolated from the left lung by expanded polytetrafluoroethylene membrane, drains are inserted, and both surgical wounds are closed.

\section{Comments}

\section{Clinical results}

Initial limited single-center analyses corroborated noninferiority of the less invasive pump implantation $(1,2)$. Consequently, encouraging clinical outcomes were demonstrated in the multicenter, prospective, nonrandomized LATERAL trial with 144 patients using the
HeartWare Ventricular Assist System (HVAD) (Medtronic, Minneapolis, MN, USA) significantly outperforming the historical sternotomy access performance goal (3). A superior one-year survival outcome with the same pump was noted in emergently implanted INTERMACS 1 profile patients compared to sternotomy by Wert et al. (4). A paucity of published evidence exists on the newest centrifugal pump HeartMate 3, yet current unpublished institutional experience is positive, as less invasive implant strategy consistently accounts for one-third of overall implants and clearly dominates reoperation cases.

\section{Advantages}

While a less invasive strategy provides potential benefits such as reduced surgical trauma and blood loss, decreased intensive care and overall length of stay, the evidence is not conclusive. Given limited pericardial cavity exposure, this specific surgical technique may also confine adhesions in prospect of subsequent transplant. A protective effect on right ventricular function by maintaining a physiological pericardial restraint is broadly discussed. Nonetheless, a need for temporary RV support has been reported for the aforementioned surgical strategy (2). Ultimately, the authors consider the most prominent advantage in previous sternotomy implants by mitigating adhesion-related injury and bleeding to heart structures (5).

\section{Caveats}

The inherent caveat to any less-invasive surgery is limited exposure and incomplete visual control of the device components (e.g., the outflow graft). Addressing any bleeding complication and anatomical injury poses a considerable challenge, thus thoughtful and highly focused surgical technique is of paramount importance. The HVAD pump may represent a surgically more appealing alternative in terms of pump and outflow graft diameter. Nonetheless, growing evidence suggests that a less invasive HeartMate 3 implant strategy is possible. Acknowledging the difficulties with the approach due to the pump profile and the minimal intercostal incision; the use of proprietary tools to facilitate the insertion (e.g., the HeartMate 3 clamp and holder Omnimedics CZ, Ltd.) appears advantageous.

\section{Acknowledgments}

Funding: None. 


\section{Footnote}

Conflicts of Interest: Dr. Netuka reports grants, personal fees and non-financial support from Abbott Int.; grants, personal fees and non-financial support from Carmat SA; non-financial support and other from LeviticusCardio Ltd. Dr. Ivak reports consulting fees from Abbott Int. and consulting fees from CARMAT SA. The other authors have no conflicts of interest to declare.

Open Access Statement: This is an Open Access article distributed in accordance with the Creative Commons Attribution-NonCommercial-NoDerivs 4.0 International License (CC BY-NC-ND 4.0), which permits the noncommercial replication and distribution of the article with the strict proviso that no changes or edits are made and the original work is properly cited (including links to both the formal publication through the relevant DOI and the license). See: https://creativecommons.org/licenses/by-nc-nd/4.0/.

Cite this article as: Netuka I, Konarik M, Pokorny M, Ivak P, Maly J, Szarszoi O. Less-invasive tools and technique for fully magnetically levitated centrifugal pump implantation. Ann Cardiothorac Surg 2021;10(2):289-291. doi:10.21037/acs-2020cfmcs-32

\section{References}

1. Mohite PN, Sabashnikov A, Raj B, et al. Minimally Invasive Left Ventricular Assist Device Implantation: A Comparative Study. Artif Organs 2018;42:1125-31.

2. Reichart D, Brand CF, Bernhardt AM, et al. Analysis of Minimally Invasive Left Thoracotomy HVAD Implantation - A Single-Center Experience. Thorac Cardiovasc Surg 2019;67:170-5.

3. McGee E Jr, Danter M, Strueber M, et al. Evaluation of a lateral thoracotomy implant approach for a centrifugalflow left ventricular assist device: The LATERAL clinical trial. J Heart Lung Transplant 2019;38:344-51.

4. Wert L, Chatterjee A, Dogan G, et al. Minimally invasive surgery improves outcome of left ventricular assist device surgery in cardiogenic shock. J Thorac Dis 2018;10:S1696-S1702.

5. Netuka I. HeartMate 3 left ventricular assist system implantation technique: the devil is in the detail. Interact Cardiovasc Thorac Surg 2018;27:946-9. 